\title{
Circular RNA cSMARCA5 regulates the progression of cervical cancer by acting as a microRNA-432 sponge
}

\author{
PING HUANG, BINGLI QI, HAIRONG YAO, LIANG ZHANG, YANYING LI and QIAN LI \\ Department of Gynecology, Cangzhou Central Hospital, Cangzhou, Hebei 061000, P.R. China
}

Received September 21, 2018; Accepted November 18, 2019

DOI: $10.3892 / \mathrm{mmr} .2020 .10910$

\begin{abstract}
Circular RNAs (circRNAs) have been shown to be involved in the development of cancer. The aim of the present study was to investigate the role of circRNA SMARCA5 (cSMARCA5) in human cervical cancer. In the present study, cSMARCA5 expression was upregulated in cervical cancer tissues and cell lines. Furthermore, the proliferation rate of cells transduced with viral plasmids expressing small interfering RNA targeting cSMARCA5 was downregulated. Bioinformatics analysis predicted that microRNA (miR)-432 targeted cSMARCA5, and miR-432 was able to interact with epidermal growth factor receptor (EGFR) by binding to its 3'-untranslated region. The expression levels of EGFR, ERK1 and ERK2 were increased in cervical cancer tissues. Furthermore, correlation analysis revealed that cSMARCA5 levels were positively correlated with ERK1 and ERK2 levels. In conclusion, the present findings suggested that cSMARCA5 may play an important role in the progression of cervical cancer via the ERK signaling pathway by modulating miR-432.
\end{abstract}

\section{Introduction}

Cervical cancer is one of the most common malignancies that occur in the cervical canal (1). It is estimated that $\sim 528,000$ cases of cervical cancer, with 266,000 mortalities, occur every year (2). Although not all of the causes of cervical cancer are known, human papillomavirus infections is considered to be the main risk factor (3). Currently, the standard tumor treatments for cancer include surgery, radiation and chemotherapy, alone or in combination (4). Although treatment strategies are improving, the 5-year survival rate for cervical cancer patients is $<40 \%$ (5). Therefore, to further study cervical cancer development at the molecular level is necessary.

Correspondence to: Dr Ping Huang, Department of Gynecology, Cangzhou Central Hospital, 16 Xinhua West Road, Cangzhou, Hebei 061000, P.R. China

E-mail: huangping_gun@163.com

Key words: circular RNA SMARCA5, microRNA-432, epidermal growth factor receptor, cervical cancer
Circular RNAs (circRNAs) are single-stranded closed ring-like non-coding RNAs, with no 5'-terminal cap or a 3'-terminal poly-A tail (6). Due to their special stable structure, circRNAs cannot be degraded by RNA exonucleases (7). circRNAs have been shown to function as competitive endogenous RNAs and microRNA (miRNA) sponges $(8,9)$. Recent studies have shown that altered circRNA levels play crucial roles in carcinogenesis $(10,11)$. Circular RNA SMARCA5 (cSMARCA5; circBase ID: hsa_circ_0001445) is a novel circRNA derived from exons 15 and 16 of the SMARCA5 gene (12). A previous study reported that overexpression of cSMARCA5 could inhibit the proliferation and metastasis of hepatocellular carcinoma cells (13). However, to the best of our knowledge, the role of cSMARCA5 in cervical cancer has not been previously investigated.

miRNAs are a class of evolutionarily conserved, short non-coding RNA molecules of 18-25 nucleotides in length (14). miRNAs can modulate gene expression by binding to the 3'-untranslated regions (3'-UTRs) of target mRNAs, resulting in repression of protein translation or mRNA degradation $(15,16)$. Previous studies have shown that miRNAs are involved in multiple cellular processes, including cell proliferation, cell cycle, apoptosis and cell differentiation (17). Abnormal expression of miRNAs has been identified in various types of malignancies (18-20). Several miRNAs have been demonstrated to be involved in cervical cancer initiation and progression (21-23). miRNA-432 (miR-432) dysregulation has been shown to be involved in the carcinogenesis of many cancers (24-26). However, a limited number of studies have reported the functional role of miR-432 in cervical cancer development.

The ERK pathway is involved in the regulation of a variety of growth and differentiation pathways through several phosphorylation cascades (27). Uncontrolled growth is a necessary step for the development of all cancers (28). In many cancer types, a defect in the mitogen-activated protein/ERK pathway is believed to contribute to uncontrolled proliferation (29). Upregulation of epidermal growth factor receptor (EGFR) is frequently detected in cervical cancer and is considered to be an independent predictor for the prognosis of cervical cancer (30).

The aim of the present study was to investigate the level of cSMARCA5 expression in human cervical cancer tissues and cell lines. In addition, the present study examined the function of cSMARCA and its underlying mechanism. 


\section{Materials and methods}

Tissue samples. The present study was approved by the Medical Ethics Committee of Cangzhou Central Hospital. All patients (18-65 years) were informed of the study and signed written informed consent. Human cervical cancer tissues and adjacent normal tissues $(\mathrm{n}=56)$ were collected from patients who visited the Cangzhou Central Hospital from January 2016 to Decembr 2017. All the specimens were immediately snap-frozen and preserved in liquid nitrogen at $-80^{\circ} \mathrm{C}$ until further use.

Cell culture and transfection. The human normal cervical epithelial cell line (Ect1/E6E7) and human cervical cancer cell lines HeLa, Ca-Ski, C-33A, and SiHa were purchased from VCANBIO Cell \& Gene Engineering Corporation, Ltd. and cultured in DMEM with 10\% FBS (both from Invitrogen; Thermo Fisher Scientific, Inc.) at $37^{\circ} \mathrm{C}$ with $5 \%$ $\mathrm{CO}_{2}$. Small interfering RNA (siRNA) targeting cSMARCA5 (si-cSMARCA5; 5'-AUUGGCGACUCAAUGGAUCAG-3'), miR-432 mimic (5'-CCUCGCGUUAUAACGUUAC-3') and their corresponding negative controls (NCs; si-NC, 5'-UUC UCCGAACGUGUCA-3'; miR-NC, 5'-UUCUCCGAACGU GUCACGUAA-3') were synthesized by Shanghai GenePharma, Co., Ltd. After culturing overnight at $37^{\circ} \mathrm{C}$, HeLa and Ca-Ski cells $\left(4 \times 10^{5}\right.$ cells/well) were transfected with si-cSMARCA5, miR-432 mimic or their parental negative controls using Lipofectamine 2000 reagent (cat. no. 11668-019; Invitrogen; Thermo Fisher Scientific, Inc.) according to the manufacturer's protocol. The of siRNAs and miRNAs was $50 \mathrm{nM}$. Subsequent experiments were performed $24 \mathrm{~h}$ after transfection.

Reverse transcription-quantitative PCR (RT-qPCR). TRIzol ${ }^{\circledR}$ reagent (Invitrogen; Thermo Fisher Scientific, Inc.) was added to the tissue and cell samples to extract total RNA. After that, total RNA was treated with RNase R (Invitrogen; Thermo Fisher Scientific, Inc.) to remove linear RNAs and enrich circRNAs. Total RNA was dissolved in RNase-free water, and the concentration was measured using a NanoDrop ${ }^{\mathrm{TM}}$ 2000 spectrophotometer. cDNA was synthesized using a TaqMan MicroRNA Reverse Transcription kit (Applied Biosystems; Thermo Fisher Scientific, Inc.) for miR-432 and a One-Step PrimerScript cDNA kit (Qiagen, Inc.) was used for cSMARCA5, EGFR, ERK1 and ERK2 using $50 \mathrm{ng}$ total RNA with the temperature protocol of: $95^{\circ} \mathrm{C}$ for $30 \mathrm{sec}$ and $60^{\circ} \mathrm{C}$ for $30 \mathrm{~min}$. RT-qPCR was performed using SYBR Green PCR Master Mix kit (Thermo Fisher Scientific, Inc.). The thermocycling conditions were as follows: Initial denaturation at $95^{\circ} \mathrm{C}$ for $5 \mathrm{~min}$; followed by 30 cycles of $95^{\circ} \mathrm{C}$ for $10 \mathrm{sec}$ and annealing at $60^{\circ} \mathrm{C}$ for $45 \mathrm{sec}$; then a final extension for 10 min at $72^{\circ} \mathrm{C}$. All primers were designed and synthesized by Shanghai GenePharma Co., Ltd. The primers were as follows: cSMARCA5 forward, 5'-GCTATCAAGCTCCATCCGCAT-3' and reverse, 5'-TAAGACGAAGCACCGGA-3'; miR-432 forward, 5'-AACGAGACGACGACAGAC-3' and reverse, 5'-CTTGGAGTAGGTCATTGGGT-3'; si-cSMARCA5 5'-CATGGTCCTCGAGGTTA-3'; si-NC 5'-UGGACAACA UGGGCUCU-3'; miR-432 mimic: 5'-AUCGAGACUACG UCUGAC-3'; miR-NC 5'-AGUGCAUGCGUACGAGCU GU-3'; EGFR forward, 5'-ATGGAATACCCTGGGTGT-3' and reverse, 5'-GGACAAGCTGGTCAAGGT-3'; ERK1 forward, 5'-CCAGTTCCGAGAATAAGCGCA-3' and reverse, 5'-CGT GTCGCCATGACACATGT-3'; ERK2 forward, 5'-TCATCC AACAGACAGACGTAGT-3' and reverse, 5'-ACCAGAGCC ATCAGACGA-3'; U6 forward, 5'-GCTCGCTTCGGCAGC ACA-3' and reverse, 5'-GAGGTATTCGCACCAGAGGA-3'; GAPDH forward, 5'-ACCACAGTCCATGCCATCCAC-3' and reverse, 5'-TCCACCACCCTGTTGCTGTA-3'. GAPDH mRNA or U6 were used as endogenous reference genes. Gene expression was determined with the $2^{-\Delta \Delta \mathrm{Cq}}$ method (31).

MTT assay. Cells $\left(3 \times 10^{3}\right)$ were seeded in 96-well plates, incubated at $37^{\circ} \mathrm{C}$ for $24 \mathrm{~h}$ and stained with $0.5 \mathrm{mg} / \mathrm{ml} \mathrm{MTT}$ at $37^{\circ} \mathrm{C}$ for $4 \mathrm{~h}$. After removal of the supernatant, DMSO was added and thoroughly mixed for $15 \mathrm{~min}$. The absorbance value in each well was measured at a wavelength of $490 \mathrm{~nm}$.

Luciferase activity assay. Circinteractome (https://circinteractome.nia.nih.gov/) and TargetScan 7.2 (http://www.targetscan. org/vert_72/) were used to predict potential targets of miR-432, and binding sites between miR-432 and cSMARCA5/EGFR. The cSMARCA5 and EGFR 3'-UTR sequences were amplified and cloned into the pGL3 vector (Promega Corporation). For reporter assays, cells were cultured in 24 -well plates at $37^{\circ} \mathrm{C}$ and cotransfected with wild type (WT)-cSMARCA5 or mutant (Mut)-cSMARCA5 (WT-EGFR or Mut EGFR) and $100 \mathrm{nM}$ miR-432 mimics or miR-NC, using Lipofectamine ${ }^{\circledR} 2000$ (Invitrogen; Thermo Fisher Scientific, Inc.). After 48 h, the luciferase activity was determined with a Dual-Luciferase Reporter System (Promega Corporation) according to the manufacturer's protocols. Firefly luciferase activity was normalized to Renilla luciferase activity using the pGL3 vector.

Cell invasion assay. For cell invasion assays, $1 \times 10^{5} \mathrm{HeLa}$ and Ca-Ski cells were resuspended in $200 \mu \mathrm{l}$ serum-free RPMI-1640 medium (Gibco; Thermo Fisher Scientific, Inc.) and then plated into the upper chambers of Transwell inserts, which were coated with Matrigel. The lower chamber was filled with culture medium supplemented with $20 \%$ FBS (Gibco; Thermo Fisher Scientific, Inc.). After $24 \mathrm{~h}$ of incubation at $37^{\circ} \mathrm{C}$, the cells on the bottom surface were fixed with $4 \%$ polyoxymethylene at room temperature for $30 \mathrm{~min}$ and stained with $0.1 \%$ crystal violet at room temperature for $20 \mathrm{~min}$. Stained cells were counted and images were captured with an Olympus BX51 light microscope (magnification, x200; Olympus Corporation).

Western blotting analysis. Proteins were extracted from cultured cells by RIPA buffer (Sigma-Aldrich; Merck KGaA) containing a mixture of protease inhibitors (100X; Beijing CoWin Biotech Co., Ltd.). Protein concentrations were quantified using a bicinchoninic acid assay (Beijing CoWin Biotech Co., Ltd.). Equal quantities of protein (30 $\mu \mathrm{g} / \mathrm{lane})$ were separated via $10 \%$ SDS-PAGE and then transferred to PVDF membranes. The membranes were blocked with 5\% skimmed milk at room temperature for $1.5 \mathrm{~h}$, followed by incubation with the following primary antibodies overnight at $4^{\circ} \mathrm{C}$ : EGFR (1:1,000; cat. no. ab32562; Abcam) and GAPDH (1:5,000; cat. no. ab185059; Abcam). Then, a horseradish peroxidase-conjugated goat anti-rabbit IgG secondary 
A

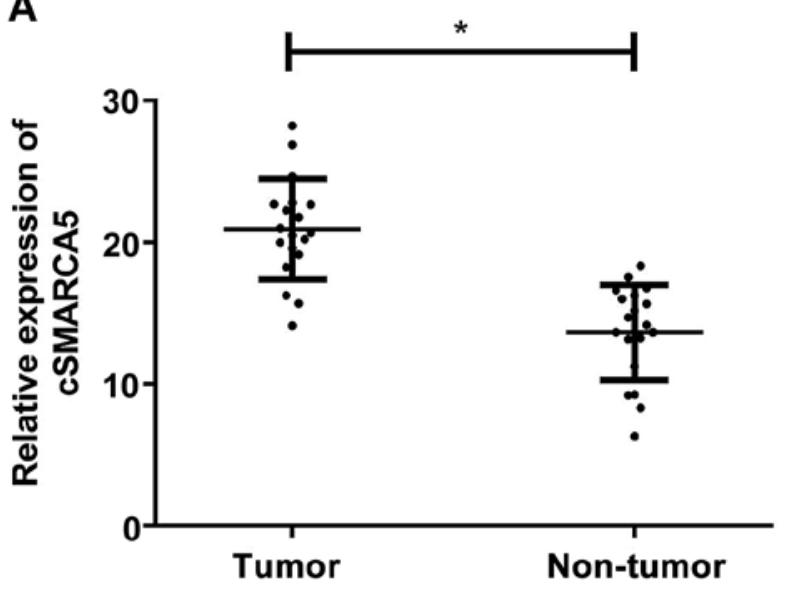

B

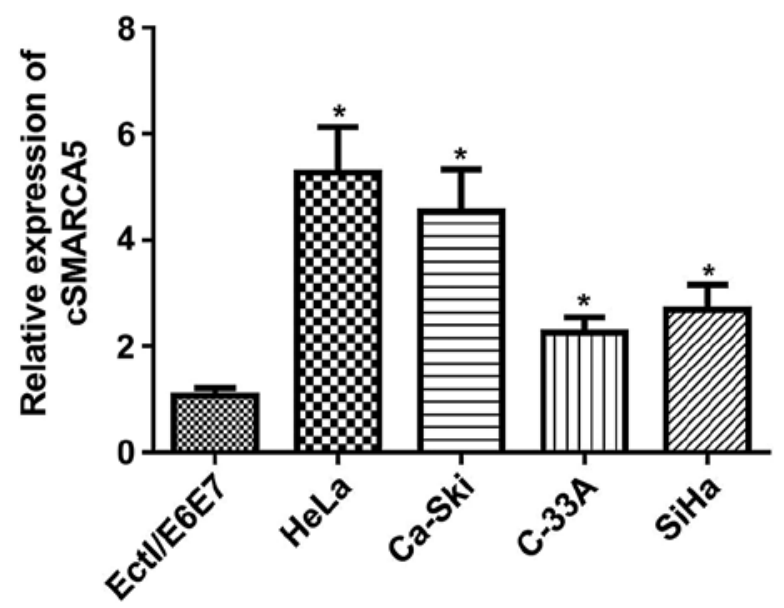

Figure 1. cSMARCA5 expression is upregulated in cervical cancer tissues and cell lines. (A) The expression level of cSMARCA5 was significantly upregulated in cervical cancer tissues compared with adjacent normal tissue samples. (B) The expression levels of cSMARCA5 were determined in the cervical cancer cell lines HeLa, C-33A, Ca-Ski and SiHa and a human normal cervical epithelial cell line Ect1/E6E7. ${ }^{\text {P }}<0.05$ vs. the respective control. cSMARCA5, circular RNA SMARCA5.

antibody (1:5,000; cat. no. sc-2054; Santa Cruz Biotechnology, Inc.) was incubated with the PVDF membranes for $1 \mathrm{~h}$ at room temperature. Protein signals were visualized using an Enhanced Chemiluminescence Plus reagent (GE Healthcare Life Sciences). Bands were quantified using Quantity One version 4.62 software (Bio-Rad Laboratories, Inc.).

Statistical analysis. Data are presented as the mean \pm SEM. All statistical analyses were performed using SPSS software (version 19.0; IBM Corp) and GraphPad Prism software (version 5.0; GraphPad Software, Inc.). Data were analyzed using a Student's t-test for two-group comparisons, and one-way ANOVA with a Tukey's post-hoc test for multiple-group comparison. Spearman's correlation analysis was used to analyze the association between cSMARCA5 and ERK1 or ERK2 expression. $\mathrm{P}<0.05$ was considered to indicate a statistically significant difference.

\section{Results}

cSMARCA5 expression is upregulated in cervical cancer tissues and cell lines. cSMARCA5 expression level in cervical cancer tissues was investigated using RT-qPCR. The expression level of cSMARCA5 was significantly increased in cervical cancer tissues (Fig. 1A) compared with non-tumor tissues. In addition, the present study examined the expression level of cSMARCA5 in four cervical cancer cell lines (HeLa, $\mathrm{Ca}-\mathrm{Ski}, \mathrm{C}-33 \mathrm{~A}$, and $\mathrm{SiHa}$ ) and a human normal cervical epithelial cell line (Ect1/E6E7). A significant increase in cSMARCA5 expression level was found in the four cancer cell lines compared with Ect1/E6E7 cells (Fig. 1B).

cSMARCA5 silencing represses the proliferation and invasion of cervical cancer cells. The oncogenic role of cSMARCA5 in cervical cancer cell lines was investigated, and it was observed that cSMARCA5 expression level was remarkably decreased in two cell lines (HeLa and Ca-Ski), which were transfected with si-cSMARCA5. The RT-qPCR results showed that cSMARCA5 expression level was significantly downregulated in the two cell lines transfected with si-cSMARCA5 (Fig. 2A and B). In addition, the MTT assay revealed that the proliferation rate of cells transduced with si-cSMARCA5 was significantly decreased at $96 \mathrm{~h}$ compared with cells transduced with si-NC in the two cell lines (Fig. 2C and D). Cell invasion assays revealed that si-cSMARCA5 suppressed the invasion of cervical cancer lines (Fig. 2E).

miR-432 targets cSMARCA5. The binding sites between miR-432 and cSMARCA5 are presented in Fig. 3A. miR-432 expression level was significantly decreased in cervical cancer tissues (Fig. 3B). miR-432 expression was significantly increased in two cell lines (HeLa and Ca-Ski) following transfection of miR-432 mimic (Fig. 3C). Decreased luciferase activity was observed in cells transfected with miR-432 mimic and cSMARCA5 wild-type reporter, but not in cells transfected with the cSMARCA5 mutant reporter plasmid and miR-432 mimic (Fig. 3D). Moreover, miR-432 expression levels were significantly increased in cells transduced with si-cSMARCA5 compared with the control group (Fig. 3E).

miR-432 targets the 3'-UTR of EGFR. Bioinformatics analysis found that the 3'UTR of EGFR may interact with miR-432. The binding site between miR-432 and EGFR is shown in Fig. 4A. The miR-432 mimic significantly reduced the relative luciferase activity of the EGFR luciferase reporter in transfected cells (Fig. 4B), suggesting a direct interaction between miR-432 and EGFR. Moreover, the miR-432 mimic significantly downregulated EGFR expression in HeLa and Ca-Ski cells at the mRNA and protein levels (Fig. 4C and D). Collectively, the present results suggested that miR-432 inhibited the expression of EGFR by binding to the 3'-UTR of EGFR.

Expression levels of EGFR, ERK1 and ERK2 in cervical cancer tissues. EGFR expression in cervical cancer tissue 
A

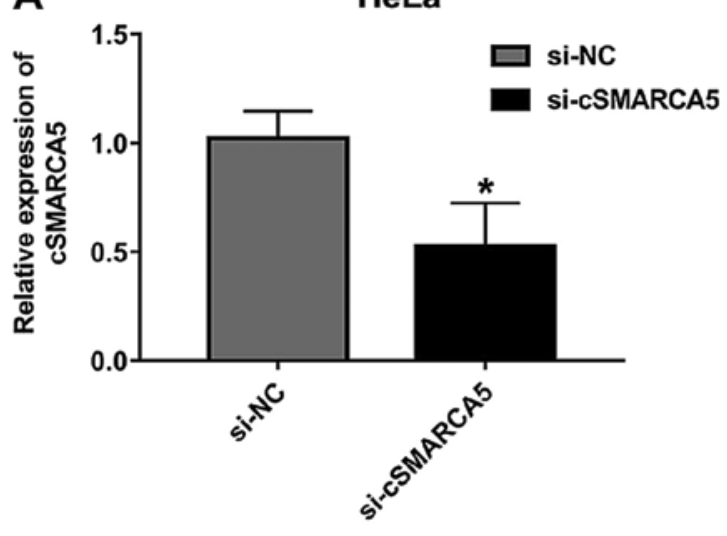

C

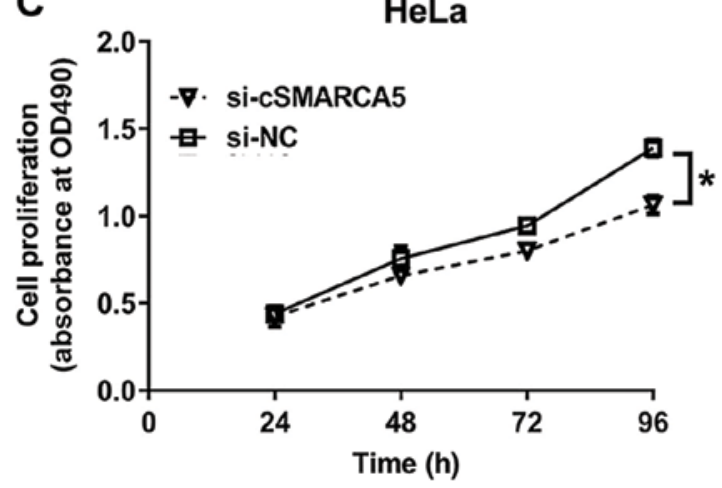

E

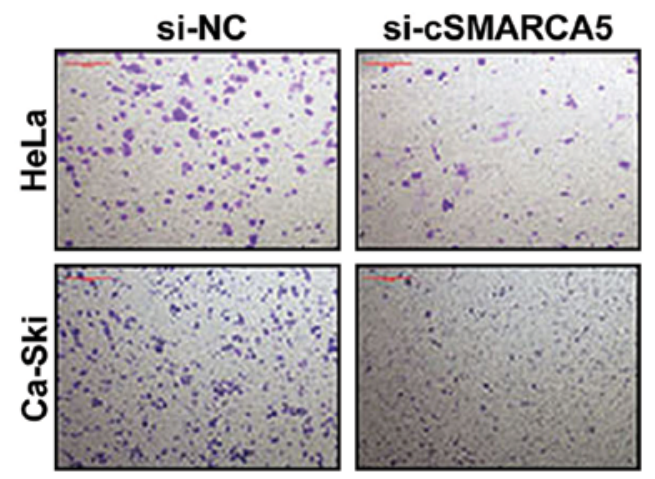

B

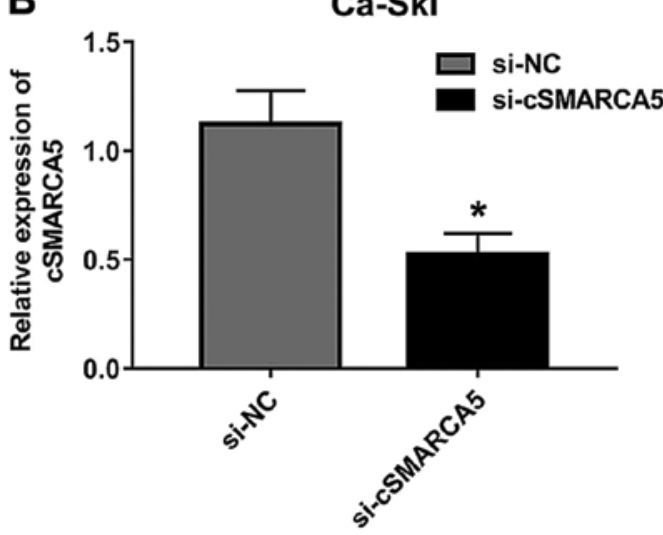

D
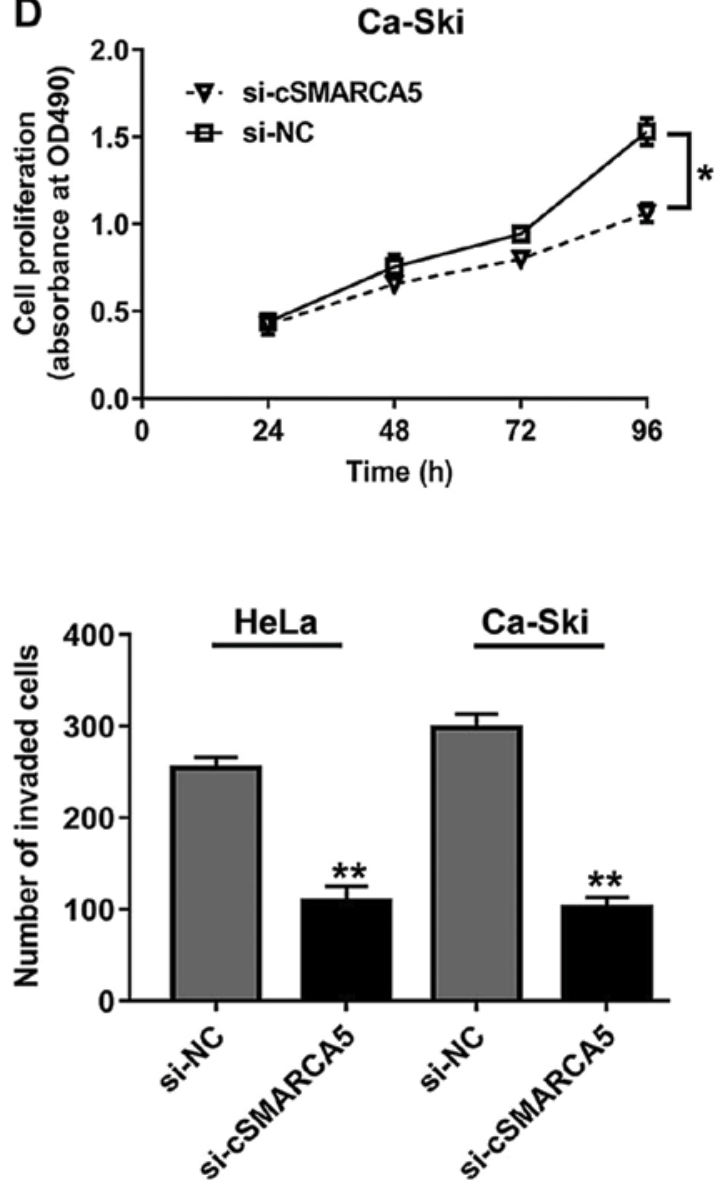

Figure 2. cSMARCA5 silencing suppresses the proliferation and invasion of cervical cancer cells. (A) cSMARCA5 expression levels were significantly downregulated in HeLa cells transduced with si-cSMARCA5 compared with si-NC. (B) cSMARCA5 expression levels were significantly downregulated in Ca-Ski cells transduced with si-cSMARCA5 compared with si-NC. (C) The proliferation rate of cells transfected with si-cSMARCA5 was significantly decreased at $96 \mathrm{~h}$ compared with cells transfected with si-NC in HeLa cells. (D) The growth rate of cells transfected with si-cSMARCA5 was significantly decreased at $96 \mathrm{~h}$ compared with cells transfected with si-NC in Ca-Ski cells. (E) Cell invasion assays revealed that si-cSMARCA5 inhibited the invasive ability of HeLa cells and Ca-Ski cells. ${ }^{*} \mathrm{P}<0.05,{ }^{* *} \mathrm{P}<0.01$ vs. respective control. Scale bar, $20 \mu \mathrm{m}$. cSMARCA5, circular RNA SMARCA5; si-NC, small interfering RNA-negative control; si-cSMARCA5, small interfering RNA-cSMARCA5.

samples was subsequently analyzed. EGFR levels were significantly increased in cervical cancer tissues compared with non-tumor tissues (Fig. 5A). The levels of ERK1 and ERK2 expression were significantly increased in cervical cancer tissues (Fig. 5B and C). Furthermore, correlation analysis revealed that cSMARCA5 levels were positively correlated with ERK1 ( $\mathrm{r}=0.962, \mathrm{P}<0.05$; Fig. 5D) and ERK2 levels $(r=0.951, P<0.05$; Fig. 5E).

\section{Discussion}

In recent years, with the rapid development of bioinformatics technology, an increasing number of circRNAs have been reported to be involved in the development and progression of various malignant tumors (32). A previous study showed that hsa_circ_0005075 is involved in cell adhesion during hepatocellular carcinoma development (33). High levels of 
A

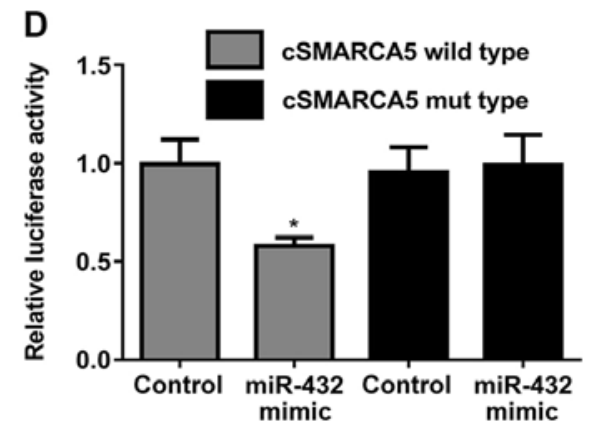

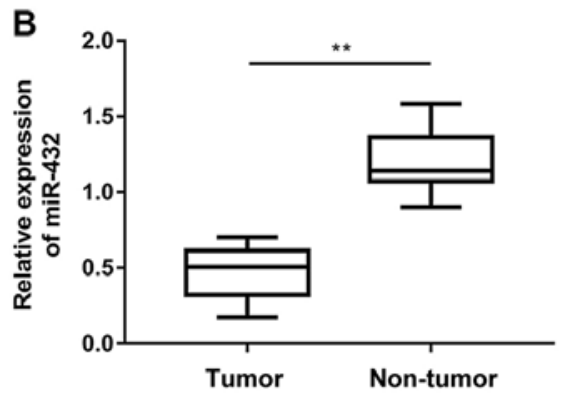
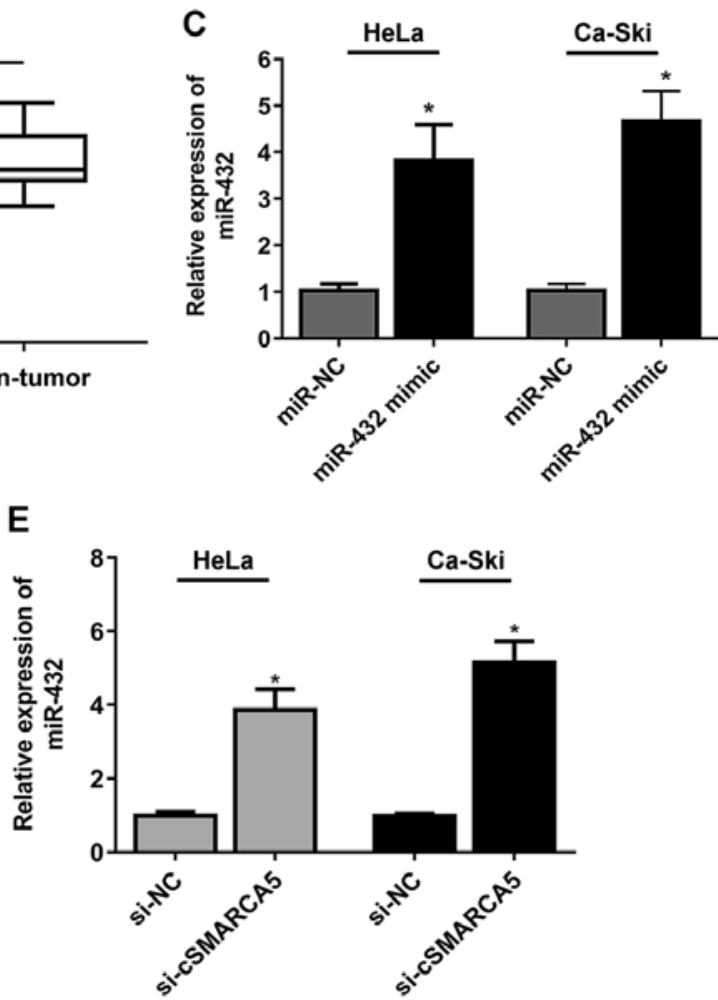

Figure 3. miR-432 targets cSMARCA5. (A) Binding sites between miR-432 and cSMARCA5. (B) miR-432 expression level was significantly decreased in cervical cancer tissues. (C) miR-432 expression level was remarkably increased in two cell lines (HeLa and Ca-Ski) that were transduced with miR-432 mimic. (D) Decreased luciferase activity was observed between cells transfected with miR-432 mimic and cSMARCA5 wild-type construct compared with cells transfected with NC-mimics. (E) miR-432 expression levels were significantly increased in cells transduced with si-cSMARCA5. ${ }^{*} \mathrm{P}<0.05,{ }^{* *} \mathrm{P}<0.01$ vs. respective control. miR-432; microRNA-432; cSMARCA5, circular RNA SMARCA5; miR-NC, microRNA-negative control; si-NC, small interfering RNA-negative control; si-cSMARCA5, small interfering RNA-cSMARCA5.

A

$$
\begin{array}{rcc}
\text { EGFR 3'UTR Wt } & 5, & \text {...GCAAAACACTAAAGATCCAAGAA... } \\
\text { miR-432 } & 3, & \text { GGTGGGTTACTGGATGAGGTTCT.. } \\
\text { EGFR 3'UTR Mut } & 5, & \text {...GCAAAACACTAAAGACTATTCCA... }
\end{array}
$$

B

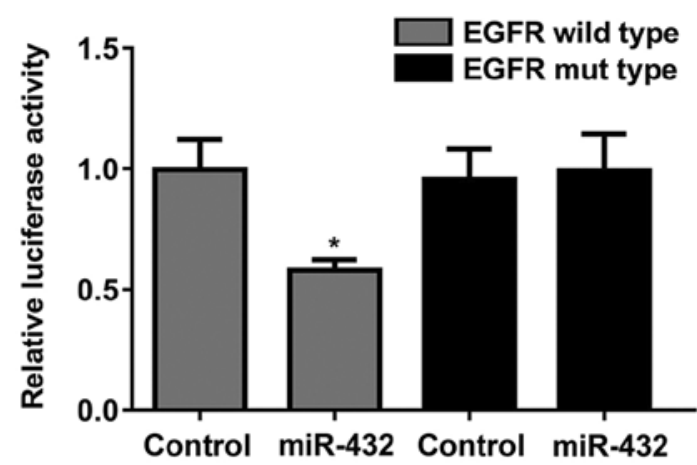

C
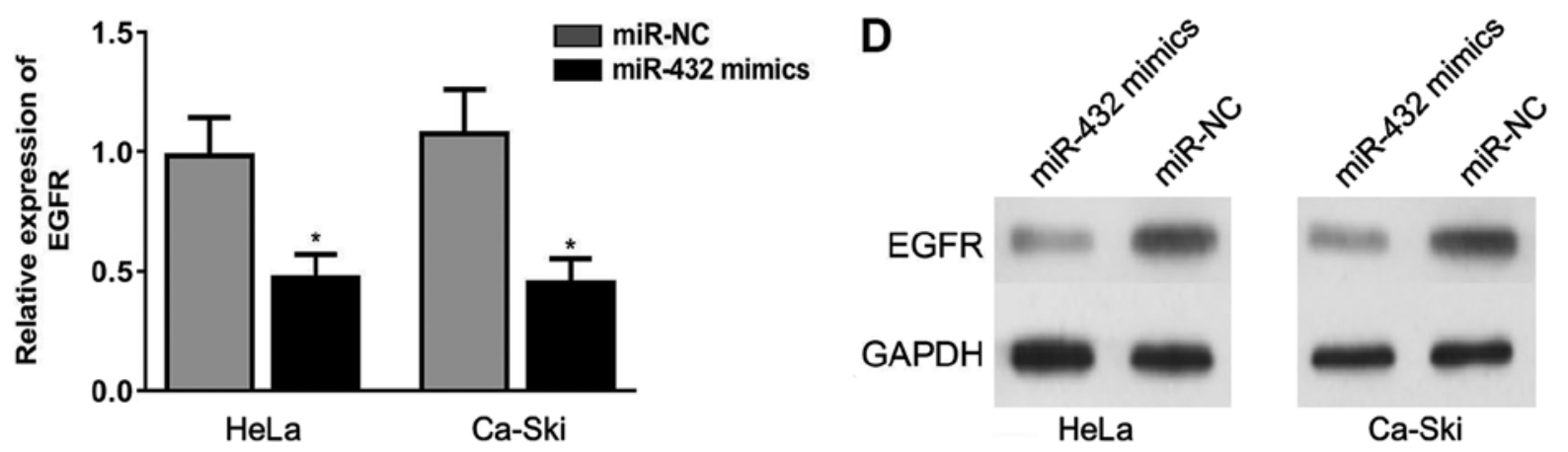

Figure 4. miR-432 targets the 3'-UTR of EGFR. (A) The 3' UTR of EGFR was found to be highly complementary to the sequence of miR-432. (B) Transfection of miR-432 mimics significantly reduced the relative luciferase activity in HeLa and Ca-Ski cells. miR-432 overexpression significantly downregulated EGFR expression in HeLa and Ca-Ski cells at the (C) mRNA and (D) protein levels. "P<0.05 vs. the respective control. miR-432; microRNA-432; miR-NC, microRNA-negative control; 3'-UTR, 3'-untranslated regions; EGFR, epidermal growth factor receptor. 
A

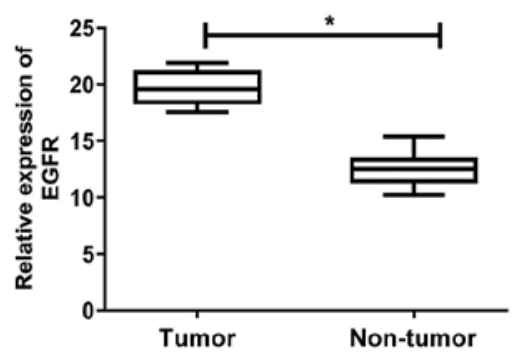

B

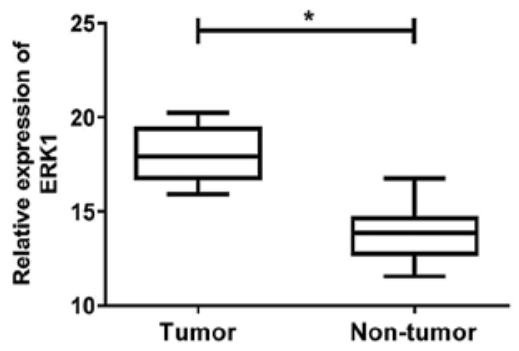

C

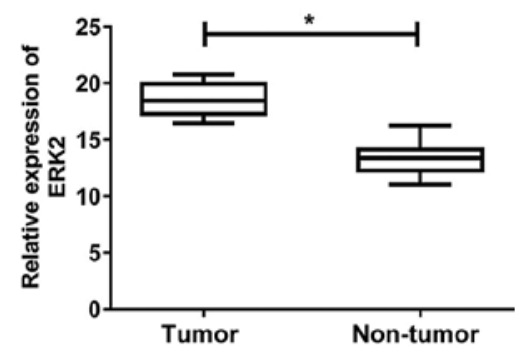

D

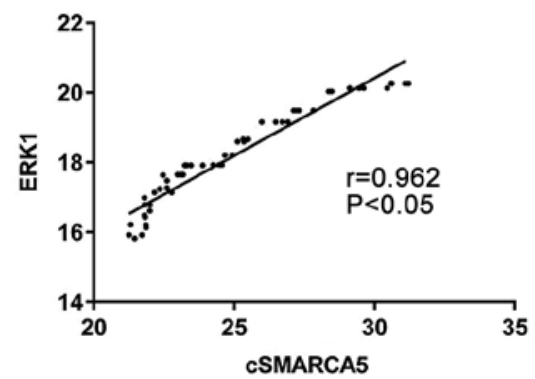

E

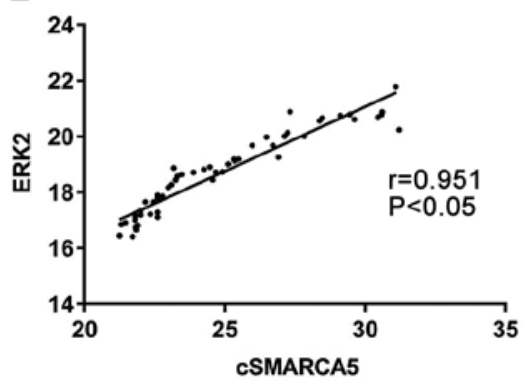

Figure 5. Expression levels of EGFR, ERK1 and ERK2 in cervical cancer tissues. (A) Expression level of EGFR was significantly upregulated in cervical cancer tissues compared with adjacent normal tissue samples. Expression levels of (B) ERK1 and (C) ERK2 were significantly increased in cervical cancer tissues. Correlation analysis revealed that cSMARCA5 levels were positively correlated with (D) ERK1 and (E) ERK2 levels. "P<0.05. EGFR, epidermal growth factor receptor; cSMARCA5, circular RNA SMARCA5.

circular RNA CCDC66 promote colorectal cancer growth and metastasis by sponging miRNAs (34). In the present study, cSMARCA5 expression level was found to be upregulated in cervical cancer tissues. In addition, a significant increase in the cSMARCA5 level in the four cell lines was observed in the present study. The proliferation and invasion of tumor cells are important characteristics that affect the progression of tumors (35). The present results suggested that cSMARCA5 could promote cervical cancer cell proliferation and invasion. In addition, results suggested that cSMARCA5 may present tumor-promoting abilities in cervical cancer cells.

It is hypothesized that circRNAs modulate diverse biological processes, acting as miRNA 'sponges', as well as regulating transcription, protein binding and translation (36). In the present study, the miRNA 'sponge' theory was explored in relation to cSMARCA5 in cervical cancer. Using bioinformatics analysis, it was predicted that miR-432 was a target of cSMARCA5. The miR-432 expression level was increased following when cSMARCA5 knockdown. The present results suggested that cSMARCA5 may induce the progression of cervical cancer by targeting miR-432.

The human EGFR gene is localized on the 7th chromosome and encodes a glycoprotein composed of $\sim 53$ amino acids that is activated by binding to specific ligands, including EGF and transforming growth factor $\alpha$ (37). Biesterfeld et al (38) found that EGFR expression was upregulated in cervical carcinomas, suggesting that the expression of EGFR may correlate with the aggressive and proliferative phenotype of cervical carcinoma. In line with previous research, in the present study, upregulation of EGFR was detected in cervical cancer tissues. Using bioinformatics analysis, the present study showed that miR-432 directly targeted the 3'-UTR of EGFR, suggesting that the miR-432 mimic could directly downregulate EGFR expression.
As a highly conserved mitogen-activated protein kinase family member, ERK plays important roles in biological processes such as proliferation, differentiation and apoptosis (39). A previous study revealed that inhibition of the ERK pathway could exert antitumor effects (40). The present results showed that ERK1 and ERK2 expression levels increased significantly in cervical cancer tissues. Furthermore, the present study found a positive correlation between cSMARCA5 and ERK1/2, suggesting that cSMARCA5 could affect the progression of cervical cancer by upregulating the ERK1/2 signaling pathway. The present study suggested that cSMARCA5 promoted the progression of cervical cancer by modulating miR-432, and induced the proliferation and invasion of cervical cancer by upregulating the ERK1/2 signaling pathway.

\section{Acknowledgements}

Not applicable.

\section{Funding}

No funding was received.

\section{Availability of data and materials}

The datasets used and/or analyzed during the current study are available from the corresponding author on reasonable request.

\section{Authors' contributions}

BQ, HY and LZ participated in data analysis and manuscript preparation. YL and QL performed the experiments and $\mathrm{PH}$ interpreted the data and drafted the manuscript. 


\section{Ethics approval and consent to participate}

The present study was approved by The Medical Ethics Committee of Cangzhou Central Hospital. All patients were informed of the study and signed written informed consent.

\section{Patient consent for publication}

Not applicable.

\section{Competing interests}

The authors declare that they have no competing interests.

\section{References}

1. Pecorelli S: Revised FIGO staging for carcinoma of the vulva, cervix, and endometrium. Int J Gynaecol Obstet 105: 103-104, 2009.

2. Ferlay J, Soerjomataram I, Dikshit R, Eser S, Mathers C, Rebelo M, Parkin DM, Forman D and Bray F: Cancer incidence and mortality worldwide: Sources, methods and major patterns in GLOBOCAN 2012. Int J Cancer 136: E359-E386, 2015.

3. Walboomers JM, Jacobs MV, Manos MM, Bosch FX, Kummer JA, Shah KV, Snijders PJ, Peto J, Meijer CJ and Muñoz N: Human papillomavirus is a necessary cause of invasive cervical cancer worldwide. J Pathol 189: 12-19, 1999.

4. Yee GP, de Souza P and Khachigian LM: Current and potential treatments for cervical cancer. Curr Cancer Drug Targets 13 205-220, 2013.

5. Smith RA, Brooks D, Cokkinides V, Saslow D and Brawley OW: Cancer screening in the United States, 2013: A review of current American Cancer Society guidelines, current issues in cancer screening, and new guidance on cervical cancer screening and lung cancer screening. CA Cancer J Clin 63: 88-105, 2013.

6. Chen LL and Yang L: Regulation of circRNA biogenesis. RNA Biol 12: 381-388, 2015.

7. Chen LL: The biogenesis and emerging roles of circular RNAs. Nat Rev Mol Cell Biol 17: 205-211, 2016.

8. Salzman J: Circular RNA expression: Its potential regulation and function. Trends Genet 32: 309-316, 2016.

9. Hansen TB, Jensen TI, Clausen BH, Bramsen JB, Finsen B, Damgaard CK and Kjems J: Natural RNA circles function as efficient microRNA sponges. Nature 495: 384-388, 2013.

10. Zheng Q, Bao C, Guo W, Li S, Chen J, Chen B, Luo Y, Lyu D, Li Y, Shi G, et al: Circular RNA profiling reveals an abundant circHIPK 3 that regulates cell growth by sponging multiple miRNAs. Nat Commun 7: 11215, 2016.

11. Pan H, Li T, Jiang Y, Pan C, Ding Y, Huang Z, Yu H and Kong D: Overexpression of circular RNA ciRS-7 abrogates the tumor suppressive effect of miR-7 on gastric cancer via PTEN/PI3K/AKT signaling pathway. J Cell Biochem 119: 440-446, 2018.

12. Glazar P, Papavasileiou P and Rajewsky N: circBase: A database for circular RNAs. RNA 20: 1666-1670, 2014.

13. Yu J, Xu QG, Wang ZG, Yang Y, Zhang L, Ma JZ, Sun SH, Yang F and Zhou WP: Circular RNA cSMARCA5 inhibits growth and metastasis in hepatocellular carcinoma. J Hepatol 68: 1214-1227, 2018.

14. Ambros V: The functions of animal microRNAs. Nature 431: 350-355, 2004.

15. Valencia-Sanchez MA, Liu J, Hannon GJ and Parker R: Control of translation and mRNA degradation by miRNAs and siRNAs. Genes Dev 20: 515-524, 2006.

16. Bartel DP: MicroRNAs: Genomics, biogenesis, mechanism, and function. Cell 116: 281-297, 2004.

17. Ebert MS and Sharp PA: Roles formicroRNAs in conferring robustness to biological processes. Cell 149: 515-524, 2012.

18. Lv D, Zhen Z and Huang D: MicroRNA-432 is downregulated in osteosarcoma and inhibits cell proliferation and invasion by directly targeting metastasis-associated in colon cancer-1. Exp Ther Med 17: 919-926, 2019.

19. Wu K, Ma L and Zhu J: miR4835p promotes growth, invasion and selfrenewal of gastric cancer stem cells by Wnt/betacatenin signaling. Mol Med Rep 14: 3421-3428, 2016.
20. Liu F, Cai Y, Rong X, Chen J, Zheng D, Chen L, Zhang J, Luo R, Zhao $\mathrm{P}$ and Ruan J: MiR-661 promotes tumor invasion and metastasis by directly inhibiting RB1 in non small cell lung cancer. Mol Cancer 16: 122, 2017.

21. Zhang X, Li F and Zhu L: Clinical significance and functions of microRNA-93/CDKN1A axis in human cervical cancer. Life Sci 209: 242-248, 2018.

22. Pedroza-Torres A, Campos-Parra AD, Millan-Catalan O, Loissell-Baltazar YA, Zamudio-Meza H, Cantú de León D, Montalvo-Esquivel G, Isla-Ortiz D, Herrera LA, ÁngelesZaragoza Ó, et al: MicroRNA-125 modulates radioresistance through targeting p21 in cervical cancer. Oncol Rep 39: 1532-1540, 2018.

23. Tan D, Zhou C, Han S, Hou X, Kang S and Zhang Y: MicroRNA-378 enhances migration and invasion in cervical cancer by directly targeting autophagy-related protein 12 . Mol Med Rep 17: 6319-6326, 2018.

24. Jiang N, Chen WJ, Zhang JW, Xu C, Zeng XC, Zhang T, Li Y and Wang GY: Downregulation of miR-432 activates Wnt/ $\beta$-catenin signaling and promotes human hepatocellular carcinoma proliferation. Oncotarget 6: 7866-7879, 2015.

25. Das E and Bhattacharyya NP: MicroRNA-432 contributes to dopamine cocktail and retinoic acid induced differentiation of human neuroblastoma cells by targeting NESTIN and RCOR1 genes. FEBS Lett 588: 1706-1714, 2014.

26. Chen L, Kong G, Zhang C, Dong H, Yang C, Song G, Guo C, Wang L and $\mathrm{Yu} \mathrm{H}$ : MicroRNA-432 functions as a tumor suppressor gene through targeting E2F3 and AXL in lung adenocarcinoma. Oncotarget 7: 20041-20053, 2016.

27. Gollob JA, Wilhelm S, Carter C and Kelley SL: Role of Raf kinase in cancer: Therapeutic potential of targeting the Raf/MEK/ERK signal transduction pathway. Semin Oncol 33: 392-406, 2006

28. Downward J: Targeting RAS signalling pathways in cancer therapy. Nat Rev Cancer 3: 11-22, 2003.

29. Leicht DT, Balan V, Kaplun A, Singh-Gupta V,Kaplun L, Dobson M and Tzivion G: Raf kinases: Function, regulation and role in human cancer. Biochim Biophys Acta 1773: 1196-1212, 2007.

30. Kersemaekers AM, Fleuren GJ, Kenter GG, Van den Broek LJ, Uljee SM, Hermans J and Van de Vijver MJ: Oncogene alterations in carcinomas of the uterine cervix: Overexpression of the epidermal growth factor receptor is associated with poor prognosis. Clin Cancer Res 5: 577-586, 1999.

31. Schmittgen TD and Livak KJ: Analyzing real-time PCR data by the comparative C(T) method. Nat Protoc 3: 1101-1108, 2008

32. Vo JN, Cieslik M, Zhang Y, Shukla S, Xiao L, Zhang Y, Wu YM, Dhanasekaran SM, Engelke CG, Cao X, et al: The landscape of circular RNA in cancer. Cell 176: 869-881.e13, 2019.

33. Shang X, Li G, Liu H, Li T, Liu J, Zhao Q and Wang C: Comprehensive circular RNA profiling reveals that hsa circ 0005075, a new circular RNA biomarker, is involved in hepatocellular crcinoma development. Medicine (Baltimore) 95: e3811, 2016.

34. Hsiao KY, Lin YC, Gupta SK, Chang N, Yen L, Sun HS and Tsai SJ: Noncoding effects of circular RNA CCDC66 promote colon cancer growth and metastasis. Cancer Res 77: 2339-2350, 2017.

35. Wang PL, Liu B, Xia Y, Pan CF, Ma T and Chen YJ: Long non-coding RNA-low expression in tumor inhibits the invasion and metastasis of esophageal squamous cell carcinoma by regulating p53 expression. Mol Med Rep 13: 3074-3082, 2016.

36. Lasda E and Parker R: Circular RNAs: Diversity of form and function. RNA 20: 1829-1842, 2014.

37. Gullick WJ, Marsden JJ, Whittle N, Ward B, Bobrow L and Waterfield MD: Expression of epidermal growth factor receptors on human cervical, ovarian, and vulval carcinomas. Cancer Res 46: 285-292, 1986.

38. Biesterfeld S, Schuh S, Muys L, Rath W, Mittermayer C and Schroder W: Absence of epidermal growth factor receptor expression in squamous cell carcinoma of the uterine cervix is an indicator of limited tumor disease. Oncol Rep 6: 205-209, 1999.

39. Yoshioka K: Scaffold proteins in mammalian MAP kinase cascades. J Biochem 135: 657-661, 2004.

40. Kohno M and Pouyssegur J: Targeting the ERK signaling pathway in cancer therapy. Ann Med 38: 200-211, 2006.

This work is licensed under a Creative Commons Attribution-NonCommercial-NoDerivatives 4.0 International (CC BY-NC-ND 4.0) License. 